\title{
A low-power, long-life and energy-saving method for cold data storage
}

\author{
Xiaoqing Jiang ${ }^{1, a}$, Qinruo Wang ${ }^{1, b}$ \\ ${ }^{1}$ School of Computer Science and Technology, Guangdong University of Technology, Guangzhou \\ 510006, China; \\ ajxq0223@163.com, bwangqr2006@gdut.edu.cn
}

Keywords: cold data, Blu-ray disc, optical disc library, low-consumption.

\begin{abstract}
As a sharp increase of global data, energy consumption of data storage is becoming more and more prominent. According to the data access frequency, about $80 \%$ of data is not be accessed. For these data, the paper proposes a low-consumption, long-life and high-security method which uses high-density, large-capacity and intelligent optical disc library as storage device and Blu-ray disc as storage medium. Optical disc library only consumes energy when it is in data recording or reading, otherwise it is in the standby state of ultra-low power consumption, which energy consumption is much lower than disk that is always in a state of high speed rotation.
\end{abstract}

\section{Introduction}

As weibo, video, voice, and picture as representative's new way of information release, our behavior, location and even physiological data have been recorded and analyzed. Data is growing at unprecedented rates around the world, and a large-scale production, sharing and application data era is coming. Now, humans are entering a new era of data information with PB as the unit [1].

The rapid growth of data led to the expansion of data storage, which resulted in data center expanding rapidly and operating costs continuing to increase. Worldwide, the digital warehouses use about 30 billion watts of electricity, roughly equivalent to the output of 30 nuclear power plants according to estimates industry experts by The New York Times. However only 6 percent to 12 percent of the electricity were used to power servers to perform computations. The rest was essentially used to keep servers idle and most of energy consumed in the disk storage [2].

So, this paper proposes a Blu-ray optical disc library storage system to reduce energy consumption in data storage and PUE (Power Usage Effectiveness) of data center.

\section{Development and advantages of optical storage}

Optical storage technology has gone through CD, DVD and BD (Blu-ray Disc). CD and DVD both use a red laser to read and write data, and the storage capacity can be up to 17GB when single plate adopts DS-DL. In contrast, Blu-ray disc uses a wavelength of 405-nanometer blue laser to read and write, and storage capacity of monolayer surface is $25 \mathrm{~GB}$. At present, there are many different kinds of Blu-ray disc, such as 50GB, 100GB, and even 300GB named Archival Disc that is launched by Sony and Panasonic and will be up to 500GB or 1TB in the future[3]. In the global optical storage market, the new generation of Blu-ray disc has a breakthrough in storage density and speed, which make it to be best optical storage technology in the era of big data.

The solid state hard disk is so expensive that it is not suitable for storing mass data, but it can be used as the system cache for hotspot data. For mass data storage, there are many different scenarios because of different characteristics of HDD, magnetic tape and disc. Compared with HDD and magnetic tape, Blu-ray optical storage has the following unique advantages.

- Green energy, low power and low cost. Whether it is HDD, tape library, or Blu-ray optical disc library, there is not much difference in the initial investment. However, in terms of energy consumption, due to the fact that the disk has always maintained a high speed rotation, so the energy consumption is relatively large. In addition, HDD and magnetic tape have to be carried out once every few years, which requires additional costs of hardware and labor. 
- Safe and reliable, anti-attack and anti-tampering. Magnetic storage and optical storage use completely different recording principle. Magnetic disk/tape applied magnetic field to magnetic material, and the magnetic material record the data through the reversal of magnetic poles, so disk and tapes are easy subject to electromagnetic influences, which result in data unreadable. In contrast, Blu-ray disc records the data by physical change to a recording layer medium, which is an irreversible process and almost impossible to tamper with the data.

- High-durability and long-term preservation of data for more than 50 years [4]. Blu-ray disc is covered with a transparent protective layer on the recording layer, which makes it resistant to changes in the external temperature, humidity and light. Accelerated testing shows that the data can be stored more than 50 years in Blu-ray optical disc at $30^{\circ} \mathrm{C}$ environment and even more than 500 years if the special recording material is used[5]. In addition, when reading the data, the disc driver does not contact the media and not cause damage to the media, so the year of reading data is far greater than HDD and tape.

So, Blu-ray storage per unit costs minimum and is the most ideal medium for long-term storage of data, which provides an ideal solution for the problem of high energy consumption.

\section{Principles of Blu-ray disc library}

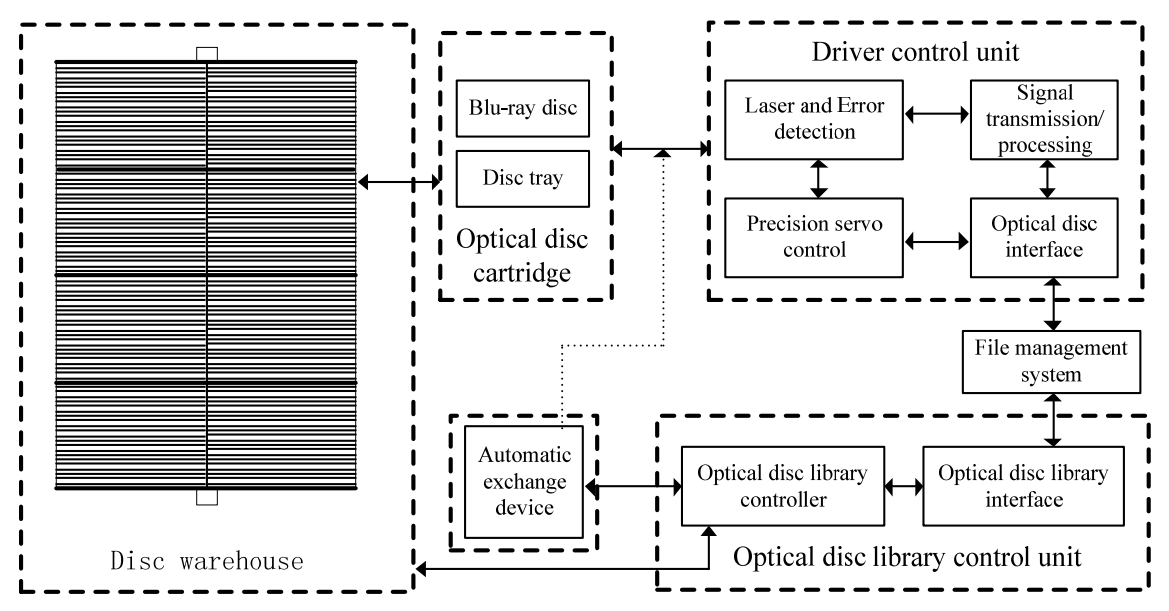

Fig. 1 Architecture of Blu-ray optical disc library

Blu-ray disc library structure as shown in Figure 1, the Blu-ray optical disc library is composed of an optical disc storage device that is a warehouse used to store Blu-ray discs, an automatic exchange device responsible for transferring the disc between optical disc storage devices and reading and writing unit of optical disc library, an optical disc library control unit responsible for scheduling of disc and allocation management of reading and writing unit, an optical disc library reading and writing unit responsible for data reading and recording and an optical disc library file management system that Organize and manage files stored in the optical disk library.

\subsection{High-density optical disc storage device}

A high-density optical disc storage device consists of a plurality of optical disk cartridges, opening and closing device, detection device, power device and controller, and optical disc cartridges are set on optical disc cartridge cage installed on the cage shaft that is supported on the supporting structure, and power device drives the cage shaft which drives the optical disc cartridge to rotate, and controller, opening and closing device and detection device are installed on the mechanical hand, which opening and closing device can guide rail movement along the manipulator with optical disc library mechanical hand and detection device is used to detect the state of opening disc cartridge. Its working principle is: when the optical disc is to be picked up, the power device drives the cartridge cage to rotate to a certain angle, and the controller controls the mechanical hand to move along the guide rail to the position of the disc cartridge, then opening and closing device opens the disc cartridge. At this time, the detecting device detects whether there is an optical disc in the opening cartridge and feedbacks the information to the controller, and then the controller controls the mechanical hand to carry out the optical disc. 
In the high-density optical disc storage device, the optical discs are stored in a stacking mode, and simultaneously, matching with the mechanical hand, the manipulator can grab more than one disc at a time. Compared with the conventional disc storage device, high-density optical disc storage device can store more discs at the same volume of space and the trip of disc cartridge automatically opening and closing is more reasonable, which improves the loading efficiency.

\subsection{Automatic exchange device}

Automatic exchange device completes the functions of picking up, transferring and separating the disc. When the disc is to be read or written, device will pick up multiple discs from optical disc cartridge and place to reading and writing unit. When the disc is to home, the device can crawl discs at one time from read-write unit and back into the corresponding disc cartridge.

Automatic exchange device consists of a mechanical hand, a guide rail, a controller, a power device, a disc separation device, etc. The guide rail is used to set the direction of the mechanical hand used to grab the multiple discs to move, and the power device drives the mechanical hand to move up and down along the guide rail. The disc separation device composed of disc jaws and disc separators can place multiple discs crawled at one time into the optical drive one by one. In the process of disc separation, disc jaws provide mechanical support to the optical discs on pickup head, and disc separators can separate the lower disc in the pickup head and the lower number of the second discs, and provide support for the optical discs on the disc separator.

Automatic exchange device can realize the functions of picking up the multiple discs at a time, picking up the multiple discs in several times, placing multiple discs at a time and placing multiple discs in several times.

\subsection{Optical disc library control unit}

Control unit is an important part of optical disc library to convert user task to the corresponding control commands and be responsible for management and allocation hardware resources which include reading and writing unit and disc storage device. Two important tables are maintained in the control unit: hardware state table of the reading and writing unit and state information table of the optical disk cartridge. When the optical disc library reading or writing, control unit will allocate the corresponding resources according to the task type and the resources state.

Task of optical disc library is stored in a list, and the control unit will pre-read the task from the list and then generate the corresponding control commands according to the task. When the task is to store data, the control unit first retrieves the disc cartridge status table to find a blank disc, while retrieving the state table of reading and writing unit to find an idle drive. The task will be performed under the circumstances that disc drive is idle and there is a blank disc, and the control unit will be called to pick up disc, and assign read-write unit. When the task is to read the data, the control unit retrieves status information from disc cartridge table according to the disc number given in read commands, then finds the given disc position in the optical disc library, and calls mechanical hand to move the disc in that condition reading and writing device is idle.

\subsection{Optical disc library file system}

An optical disc library has tens of thousands discs, and can not guarantee all discs online. So, this paper has developed a file system to organize and manage the files in optical discs.

File system of optical disc library solved the problem of access to files. After a file burned, the file system will create a file node that includes a relative path to the file in the optical disc library, disc number and other information. When a user accesses a file, the file system first detects the file state. If the file is online, the file system opens the file directly. Otherwise, the file system will retrieve the list of file nodes according to the relative path, and query the disc number, and then send disc number to control unit through the socket communication. After the specified disc is placed in the disc driver, the file system will open a file, and then the user can access the file.

\subsection{Blu-ray disc library application}

Compared with the original jukebox, optical disc library in this paper uses the file system to manage files, which makes the number of discs no longer limited by the disc management software. In this paper, the number of discs can reach up to 12000 and the storage capacity can reach PB. Above all, standby power is less than $200 \mathrm{~W}$, and the data transmission rate can reach $1152 \mathrm{MB} / \mathrm{s}$. At 
present, optical disc library has been applied to the data center of Meteorological Bureau, Seismological Bureau, archives, etc.

The method for cold data moved from RAID to optical disc library is more energy-efficient than only used RAID, and it makes a great contribution to reduce operating costs of the data center.

\section{Summary}

This paper describes an energy-saving method, which uses Blu-ray disc with advantages of low-power, long-life and high-security, to store cold data accessed hardly in optical disc library according to the data access frequency. With the advent of big data, it is the appropriate solutions to store archive and backup data in large-capacity intelligent Blu-ray disc library. Most of all, from the long-term interest of storage data, Blu-ray disc library has lowest total cost of ownership and makes an important positive role in the construction of green energy-saving for data center.

\section{References}

[1]. Barabási A L. Bursts: the hidden patterns behind everything we do, from your e-mail to bloody crusades[M]. Penguin, 2010.

[2]. Times N Y. Power, pollution and the Internet[J]. [2012-10-02]. http://www.nytimes.com/2012/ 09/23/technology/data-centers-waste-vast-amounts-of-energy-belying-industry-image.html.

[3]. Hao R, Bu C. Multilayer optical storage for big data center: by pre-layered scheme[C]// ISPDI 2013 - Fifth International Symposium on Photoelectronic Detection and Imaging. 2013:1-15.

[4]. Habuta H, Tomiyama M, Takahashi K, et al. Quadruple-Layer Write-Once Disk for Blue Laser Based on Te-O-Pd Recording Films[J]. Japanese Journal of Applied Physics, 2008, 47(9R): 7160.

[5]. Miyagawa N, Kitaura H, Takahashi K, et al. Over 500 Years Lifetime Dual-Layer Blu-Ray Disc Recordable based on Te-O-Pd Recording Material[C]// Optical Data Storage Topical Meeting, 2006. 2006:249 - 251. 